\title{
Diabetic nephropathy: evidence for renoprotection and practice
}

\author{
Carl-Erik Mogensen
}

Department of

Endocrinology and

Diabetes, Århus

Kommune Hospital,

Århus University of

Hospital,

Nørrebrogade,

DK-8000 Århus C,

Denmark

C-E Mogensen

Correspondence to: Professor Mogensen email: cem@afdm.au.dk

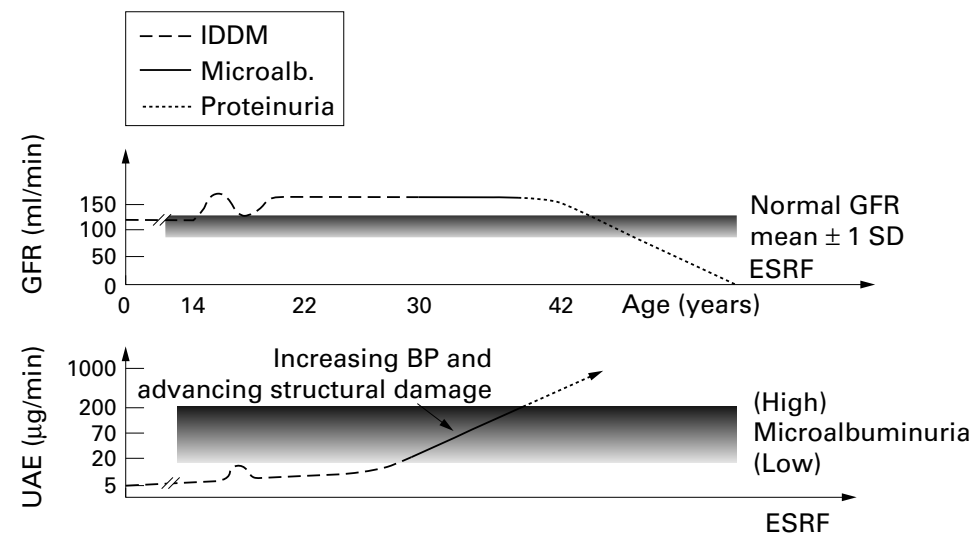

Figure 1 The natural course of diabetic nephropathy in type 1 diabetes.

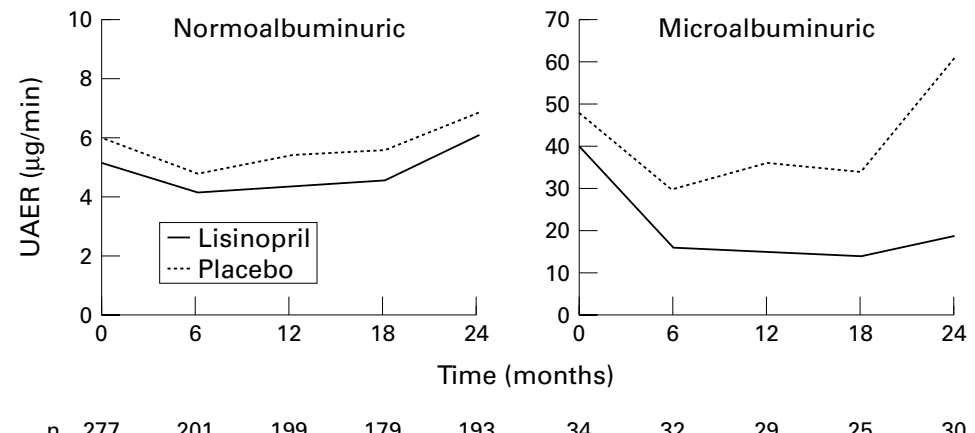

Figure 2 Changes in albumin excretion rate by initial microalbuminuria status (from the EUCLID study). microalbuminuria there is advanced structural damage in the kidney.

Later, patients develop overt nephropathy and in type 1 and type 2 diabetes there is a relentless decline in glomerular filtration rate (GFR).

\section{Role of angiotensin converting enzyme inhibitors}

In recent years there has been an increased focus on treating patients early to prevent
Patients with diabetic renal disease have a very bad prognosis. Hyperfiltration, related to poor metabolic control, occurs early on in the disease process. ${ }^{1}$ There is also an increase in albumin excretion which can be readily reversed by effective glycaemic control at clinical diagnosis (fig 1).

A phase is then entered during which most patients are normoalbuminuric. However, after approximately 10 years, some patients will develop microalbuminuria. The risk of progression from normal to microalbuminuria is closely related to poor metabolic control and also, to some extent, blood pressure elevation. In patients with type 1 or type 2 diabetes and \section{2).}

future renal damage. Even before microalbuminuria has developed there is evidence of the benefits of angiotensin converting enzyme (ACE) inhibitors.

NORMAL ALBUMINURIA

In studies looking at renal haemodynamics, ACE inhibitors were used to treat patients with type 1 diabetes who had normal albumin excretion. ${ }^{2}$ A reduction in filtration fraction was observed with ACE inhibitor treatment suggesting that the pressure at the glomerular membrane was reduced. The albumin excretion rate was also reduced in these completely normo-albuminuric patients, suggesting that by reducing pressure, albuminuria can be reduced.

MICROALBUMINURIA

Many studies document the effectiveness of ACE inhibitors in reducing microalbuminuria. In the EUCLID study, treatment of patients with type 1 diabetes with an ACE inhibitor did not produce a significant reduction in albumin excretion rate (AER) in patients with normal albuminuria. ${ }^{3}$ However, in patients with microalbuminuria there was a very significant drop in AER which was sustained over two years (fig 2).

There are now approximately 10 studies in patients with type 1 diabetes and microalbuminuria comprising 800 patients. All of these studies show a reduction in microalbuminuria with early ACE inhibition and in many of these studies blood pressure was completely normal. However, microalbuminuria is only a surrogate end point and so stronger markers for renal disease are needed.

\section{GLOMERULAR FILTRATION RATE}

GFR is an important parameter for measuring renal function. However, to consider a treatment effect on GFR in patients with microalbuminuria long term studies are needed.

In a randomised study looking at changes in GFR in diabetic patients over eight years, those treated with ACE inhibitors had a stable GFR. ${ }^{4}$ In contrast, those patients receiving placebo had a dramatic decline in GFR. This provides important evidence of a renopreservation or glomerular preservation effect of ACE inhibitors in patients with microalbuminuria.

\section{GLOMERULOPATHY}

Structural damage to the kidney should also be considered. During the typical course of diabetic renal disease, there is a progression in glomerulopathy over two to three years.

In a two and half year study there was a significant increase in glomerulopathy index in 


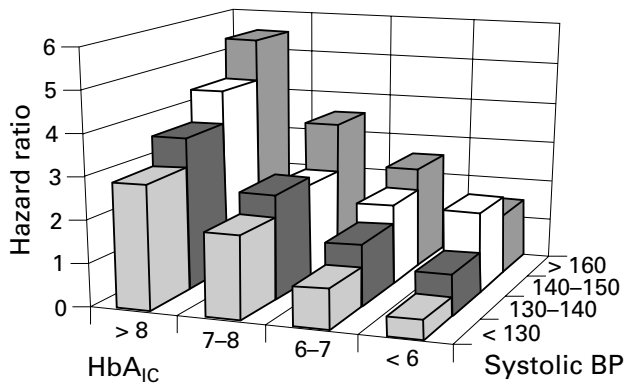

Figure 3 Impact of glycaemic and blood pressure control on diabetes related end points (from UKPDS).

the placebo group. ${ }^{5}$ In contrast, the two treatment groups-one treated with ACE inhibitors and the other treated with $\beta$ blockers - had stabilisation of glomerular damage. This provides valuable evidence that, with early antihypertensive treatment, there is preservation of renal structure, which supports previous studies showing preservation of GFR.

\section{Combination therapy}

Patients with diabetic nephropathy or microalbuminuria may have to be treated with combination therapy. Interestingly, a study looking at patients treated with $\beta$ blockers and diuretics initially, with ACE inhibitors added, showed a significant reduction in microalbuminuria and stable GFR. ${ }^{6}$

Comparison with calcium channel blocker In a one year study comparing the effects of nifedipine and lisinopril in type 2 hypertensive patients with microalbuminuria, there was a profound drop in urinary albumin excretion with the ACE inhibitor. ${ }^{7}$ In contrast, with nifedipine there was an initial drop in microalbuminuria, which then stabilised. There was a significant difference between the two groups.

UKPDS

The important UKPDS study compared the outcome of tight and less tight blood pressure control on a number of different diabetes related end points. ${ }^{8}$ There was a significant difference in blood pressure of $10 \mathrm{~mm} \mathrm{Hg}$ systolic and $5 \mathrm{~mm} \mathrm{Hg}$ diastolic between the two groups. There was also a significant effect on all diabetes related end points $(p=0.005)$. The impact of blood pressure control was earlier and more dramatic than the effect of glycaemic control.

It is hoped that UKPDS will eventually provide useful data on renal disease. So far, it has shown that early antihypertensive treatment has a positive effect on the development of microalbuminuria. However, the study has not been running for a sufficient length of time to show an effect on proteinuria. Moreover, as the development of overt nephropathy takes decades, useful data will not be available for some time.

Epidemiological data from UKPDS shows that by combining effective glycaemic and blood pressure control, the risk of any diabetes related end point is very low (fig 3).
Trial acronyms

AIPRI: Angiotensin-converting enzyme Inhibition in Progressive Renal Insufficiency

EUCLID: EUrodiab Controlled trial of Lisinopril in Insulin dependent Diabetes mellitus

UKPDS: United Kingdom Prospective Diabetes Study

ADVANCED RENAL DISEASE

In the AIPRI study, which examined the effect of the ACE inhibitor benazepril in 583 patients with various renal diseases, the proportion of patients developing advanced renal disease, or a doubling of serum creatinine, was significantly lower in patients treated with the ACE inhibitor compared with those receiving placebo. ${ }^{9}$

\section{New treatment strategies}

Increasingly attention is focusing on newer treatment strategies including the use of angiotensin II type I receptor antagonists. A study from Finland examined the potential effect of candesartan on microalbuminuria in 35 type 2 hypertensive diabetics over a 12 week period. ${ }^{10}$ Results indicate there was a decline in microalbuminuria with candesartan $(\mathrm{p}=0.03$ $v$ placebo) and a greater reduction in blood pressure than with placebo. A combination of lisinopril and candesartan has recently produced positive results.

\section{Renoprotection}

Studies undertaken in patients with type 1 and 2 diabetes have shown that with effective ACE inhibitor treatment it is possible to decrease microalbuminuria and in some cases obtain normal albuminuria. It is also possible in many patients to prevent the development of proteinuria and the fall in GFR as well as structural damage to the kidney. In conclusion, it appears that effective treatment with ACE inhibitors can prevent or postpone advanced nephropathy in patients with type 1 and 2 diabetes and postpone end stage renal disease.

\section{Summary}

Early treatment of patients with microalbuminuria with ACE inhibitors may prevent the development of more advanced disease, including cardiovascular mortality. However, it is clear that for effective management of patients with type 1 and 2 diabetes effective glycaemic and blood pressure control must be achieved.

A decline in GFR can also be prevented by more effective intervention in patients with microalbuminuria. Indeed, even in overt renal disease it is possible to change the relentless decline in GFR by antihypertensive treatment. Dual blockade of the renin-angiotensin system seems a promising treatment strategy. ${ }^{11}{ }^{12}$ 1 Mogensen CE. Prediction of clinical diabetic nephropathy
in IDDM patients. Alternatives to microalbuminuria? Diabetes 1990;39:761-7. 
2 Mau Pedersen M, Schmitz A, Pedersen EB, et al. Acute and long-term renal effects of angiotensin converting enzyme inhibition in normotensive, normoalbuminuric insulin-

dependent diabetic patients. Diabetic Med 1988,5:562-9

3 The EUCLID Study Group. Randomised placebocontrolled trial of lisinopril in normotensive patients with insulin -dependent diabetes and normoalbuminuria or microalbuminuria. Lancet 1997;349:1787-92

4 Mathiesen ER, Hommel E, Hansen HP, et al. Randomised controlled trial of long-term efficacy of captopril on preservation of kidney function in normotensive patients with insulin dependent diabetes and microalbuminuria. $B M \mathcal{F}$ $1999 ; 319 ; 24-5$.

5 Rudberg S, Østerby R, Bangstad HJ, et al. Effect of angiotensin converting enzyme inhibitor or beta-blocker on glomerular structural changes in young microalbuminuric (a) Diabetologia 1999;42:589-95.

6 Mau Pedersen M, Hansen KW, Schmitz A, et al. Effects of ACE inhibition supplementary to beta-blockers and diuretics in early diabetic nephropathy. Kidney Int 1992;41: diuretics

7 Agardh CD, Carcia-Puig J, Charbonnel B, et al. Greater reduction of urinary albumin excretion in hypertensive type II diabetic patients with incipient nephropathy by lisinopril than by nifedipine. 7 Human Hypertens 1996;10: nopril thar $185-92$.

8 Mogensen CE. Combined high blood pressure and glucose in type 2 diabetes: double jeopardy. BMF 1998;317:693-4. 9 Maschio G, Alberti D, Janin G, et al. Effect of the angiotensin-converting enzyme inhibitor benazepril on the progression of chronic renal insufficiency. The angiotensinconverting enzyme inhibition in progressive renal insufficiency study group. $N$ Engl f Med 1996;334:939-45.

10 Forsblom C, Trenkwalder P, Dahl K, et al. Candesartan cilexetil, a novel angiotensin II antagonist, reduces microalbuminuria in a patients with type II diabetes mellitus and mild hypertension. Am $\mathcal{F}$ Hypertens $1997 ; 10(\mathrm{pt}$ 2):86.

11 Mogensen CE, Neldam S, Tikkanen I, et al. Randomised controlled trial of dual constem dependent diabetes: the candesartan and lisinopril miroalbuminuria (CALM) study. BMFIn press.

12 Mogensen CE. Microalbuminuria, blood pressure and diabetic renal disease: Origin and development of ideas. In: Mogensen CE, ed. The kidney and hypertension in diabetes mellitus. Kluwer Academic Publishers, 2000:655-706 